\title{
D Outcome of Cytomegalovirus Colitis in Inflammatory Bowel Disease with Different Regimes of Ganciclovir
}

\author{
Iftikhar Ahmed ${ }^{1,2, *}$, Wael Kassem ${ }^{2}$, Yazen Salam ${ }^{3}$, Manuele Furnari ${ }^{4}$, Tina Mehta ${ }^{5}$
}

1. School of Medicine, University of Southampton, UK

2. Department of Medicine, Aldara Hospital and Medical Centre, Riyadh, KSA

3. School of Medicine, Alfaisal University, Riyadh, KSA

4. Department of Medicine, University of Genova, Italy

5. Department of Gastroenterology, Royal United Hospital Bath, UK

\section{* Corresponding Author:}

Iftikhar Ahmed, MD

Department of Medicine, Aldara Hospital and Medical Center, Riyadh, KSA, School of Medicine, University of Southampton, Southampton, UK, Yardville Compound | P.O. Box 1105 - Riyadh 11431

Tel: + 966114207845

Fax: + 966112255696

Email: iahmed@aldaramed.com

Received: 24 Apr. 2018

Accepted: 02 Sep. 2018

\section{ABSTRACT}

\section{BACKGROUND}

Cytomegalovirus (CMV) infection is common in individuals with inflammatory bowel disease (IBD) and is responsible for relapse, increased severity, and poor outcome if left untreated. Ganciclovir is the mainstay of treatment but data regarding its use, mode of administration, and duration of treatment is poorly described. We reviewed the practice of treating CMV colitis with different regimes of ganciclovir at a district NHS hospital to compare the clinical outcome.

\section{METHODS}

35 patients with IBD and concurrent diagnosis of CMV infection were evaluated. The parameters studied were clinical outcome in term of clinical response, length of hospital stay, readmission, or colectomy with three different regimes of ganciclovir, in addition to treatment for IBD.

\section{RESULTS}

35 patients with IBD (ulcerative colitis $=23$, Crohn's disease $=5$, Indeterminate colitis $=7$ ) and positive diagnosis of CMV infection were studied. Clinical outcome with two weeks of intravenous (IV) ganciclovir regime was superior than one week of IV ganciclovir and two weeks of oral Valganciclovir in term of clinical response on day $15(95.8 \%$ vs $74 \%, 24.3 \%$, respectively $p=0.45)$ and colectomy rate within 3 months ( $6.25 \%$ vs $27.3 \%$, vs $25 \%$, respectively).

\section{CONCLUSION}

CMV colitis is associated with poor outcome in patient with IBD if left untreated. 2 weeks IV ganciclovir was associated with a better outcome than 1 week of IV treatment or oral treatment.

\section{KEYWORDS:}

Cytomegalovirus colitis, Inflammatory bowel disease, Indeterminate colitis, Ganciclovir

Please cite this paper as:

Ahmed I, Kassem W, Salam Y, Furnari M, Mehta T. Outcome of Cytomegalovirus Colitis in Inflammatory Bowel Disease with Different Regimes of Ganciclovir. Middle East $J$ Dig Dis 2018;10:220-229. doi: 10.15171/mejdd.2018.114.

\section{INTRODUCTION}

Cytomegalovirus (CMV) belongs to Herpesviridae family, a double-stranded DNA virus with an outer coat made of protein and an envelope of lipoprotein. ${ }^{1}$ CMV infection is fairly common in general population, which starts off with flu-like symptoms or asymptomatic presentation but carries a risk of prolonged latency. After the initial phase, the virus has the ability to remain dormant in different types of host cells in human organs including the colon. Immunocompromised conditions can lead to viral reactivation, replication, and the initiation of a systemic disease process, which may result in various deadly 
complications including severe colitis, ${ }^{2,3}$

Inflammatory bowel disease (BD) is an immunocompromised condition. Additionally, patients are often treated with immunosuppressive medications. Both of which put such patients at a higher risk of CMV infection., CMV infection is commonly described in patients with IBD and is considered to be responsible for the disease relapse, increased severity of the disease, and poor outcome if left untreated. ${ }^{6,7}$ Although both Crohn's disease (CD) and ulcerative colitis (UC) have propensity to develop CMV infection, it has been shown that the infection is more prevalent in UC than CD for reasons not completely understood. ${ }^{8}$ This higher infection rate is noticed in those who have a latent CMV infection as the immunocompromised state of the patient leads to a higher reactivation rates. This is particularly important during the phase of disease activation and flare-ups, when these patients often become seropositive for CMV. ${ }^{9}$ The diagnosis of CMV infection is usually made by the combination of CMV antigenemia assay, detection of inclusion bodies in the colonic biopsy specimens, and or immunohistochemistry using anti-CMV monoclonal antibodies. ${ }^{10}$

Due to the severity of CMV infection and its long-term complications, there has always been a need for early diagnosis and proactive treatment. The treatment has various approaches that range from the use of anti-viral therapy to the use of CMV immunoglobulins. ${ }^{11,12}$ Ganciclovir is the mainstay of treatment due to its favorable outcome and a remission rate of $67-100 \% .^{13,14}$ Data regarding its use, mode of administration, and duration of treatment is poorly described in the literature. Foscarnet has also been shown of proven efficacy in those patients who cannot tolerate ganciclovir or if ganciclovir is contraindicated..$^{15}$

In this study, we looked at our patient population retrospectively to review the treatment of CMV colitis with ganciclovir therapy and the clinical outcome associated with three different regimes used as treatment in our IBD population.

\section{MATERIALS AND METHODS}

We conducted a retrospective review of the medical records of patients at our institution with a diagnosis of IBD who were concurrently tested positive for CMV colitis between 1st January 2005 and 1st August 2011.
Non-systematic strategy was used to test all patients who received treatment for $\mathrm{CMV}$ colitis with ganciclovir. Medical records were also electronically searched for related details. All the patients included in the analysis had provided verbal authorization for medical record review for research purposes.

\section{Inclusion criteria}

All patients aged 18 years or above with a diagnosis of $\mathrm{CD}, \mathrm{UC}$, or indeterminate colitis (IC) who were positive for CMV serology, viremia on polymerase chain reaction (PCR), and or a positive histology for CMV inclusion bodies were included in the study. All patients included were treated with ganciclovir.

\section{Exclusion criteria}

This was limited to the absence of underlying diagnosis of IBD, concurrent diagnosis of enteric infections and or positive faecal test for clostridium difficile infection (CDI), diagnosis of CMV in post-surgical specimen, loss of follow up, or age less than 18 years.

\section{Measures and analyses}

Demographic and clinical characteristics were obtained from the electronic medical record in those patients who met entry criteria. Characteristics included age, sex, smoking status, type of IBD, anatomic distribution, duration of disease, previous surgery, prior and concurrent treatment for IBD, date of CMV diagnosis, duration and mode of treatment with ganciclovir, clinical response as recorded in the medical notes, CRP level pre-treatment and on days $3^{\text {rd }}, 5^{\text {th }}, 7^{\text {th }}, 10^{\text {th }}$, and $14^{\text {th }}$ and on one month after treatment when available, CMV PCR evaluation pre- and post-treatment, detection of clostridium difficile toxins (CDT), acute or delayed hypersensitivity reactions, autoimmune reaction, and outcome in term of colectomy or clinical improvement to be discharged from hospitals. Clinical response in term of improvement in the frequency of diarrhea, rectal bleeding, and abdominal pain were retrospectively determined from the medical records. In patients with $\mathrm{CD}$, complete response was defined as cessation of diarrhea and abdominal cramping. Partial response was defined as a reduction in the amount of diarrhea and abdominal cramping. Outcomes not meeting one of the above definitions were classified as non-response. 


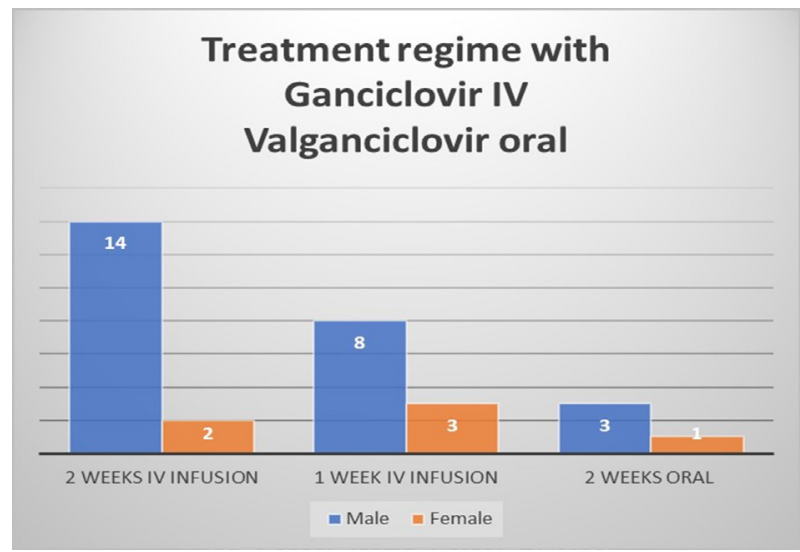

Fig.1: Graph showing demographic characteristics

In patients with UC, complete response was defined as cessation of diarrhea, hematochezia, and abdominal cramping whereas partial response was defined as a reduction in the amount of diarrhea, hematochezia, and abdominal cramping. The results of radiological and/or endoscopic imaging were documented where available. Symptoms scores of the patients were calculated using Harvey Bradshaw Index (HBI) in case of CD and Simple Colitis Clinical Activity Index (SCCAI) in case of UC and IC using the information from the patients' notes and other clinical records. The treatment regime of ganciclovir used to treat CMV colitis with duration and mode of treatment were obtained from the medical notes and pharmacy electronic record. A total of 35 patients were identified who fulfilled the inclusion criteria as above. 31 out of the 35 patients were treated with ganciclovir with three different regimes and were grouped into three categories based on the treatment regime. Group 1: those who received 2 weeks of intravenous (IV) ganciclovir $5 \mathrm{mg} / \mathrm{kg}$ twice a day, group 2: those who received one week IV ganciclovir $5 \mathrm{mg} / \mathrm{kg}$ twice a day, and group 3: those who received two weeks oral valganciclovir 900 mg twice a day.

\section{Ethics}

The study was approved by the local Research and Ethics Committee.

\section{Statistical analysis}

Descriptive statistics were used to analyze baseline characteristics. Fisher's exact test, $\chi 2$ test, and log-rank

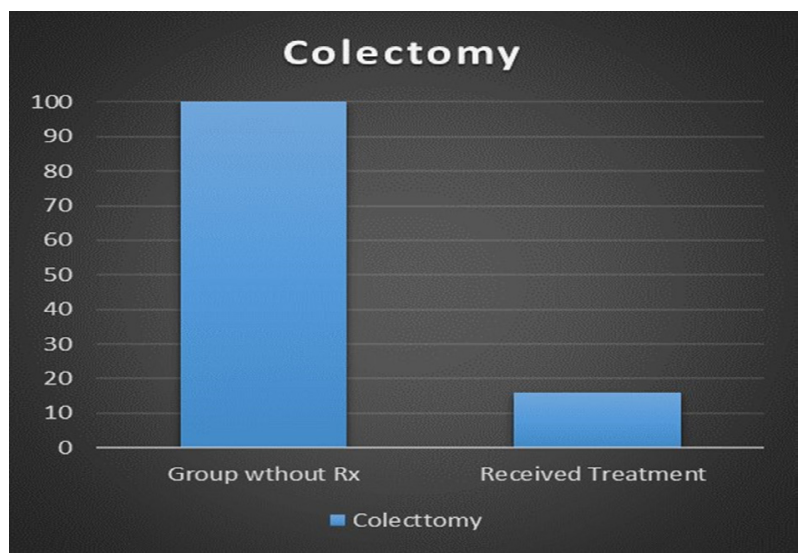

Fig.2: Graph showing colectomy rate with or without treatment with Ganciclovir

test for discontinuation were used for statistical analysis between the groups. $P$ value of 0.05 was considered as statistically significant. Statistical analyses were conducted using Statistical Analysis Software (SAS).

\section{RESULTS}

35 patients with pre-existing diagnosis of $\mathrm{IBD}(\mathrm{UC}=23$, $\mathrm{CD}=5, \mathrm{IC}=7$ ) were identified with a diagnosis of $\mathrm{CMV}$ infection between 2005 and 2011. The diagnosis of CMV infection was confirmed by detection of inclusion bodies in biopsy specimens from the colonic mucosa by either hematoxylin and eosin (H\&E) staining, or immunohistochemistry (IHC) using anti-CMV monoclonal antibodies. In most of the cases, CMV viral status was also confirmed by PCR. The age range of the patients was 33-89 years $($ mean $=51, F=14)$. All 35 patients were admitted to hospital with severe diarrhea. Four out of the 35 patients were excluded from the study. One of those excluded was admitted with severe diarrhea with initial diagnosis of IBD, which was later turned out to be ischemic colitis. The other three patients required colectomy within 5 days of admission for severe UC, after failing medical treatment with IV steroids and no treatment with ganciclovir. The diagnosis of CMV in these three cases was made by histological examination of the colectomy specimens. All the remaining 31 patients were treated with ganciclovir: 16 patients (group 1) with 2 weeks of IV ganciclovir $5 \mathrm{mg}$ / $\mathrm{kg}$ twice a day, 11 patients (group 2) with one week of IV ganciclovir $5 \mathrm{mg} / \mathrm{kg}$ twice a day, and 4 patients (group 3) with two weeks of oral valganciclovir $900 \mathrm{mg}$ twice a day treatment regime as shown in figure 1. Details of the 
Table 1: Patients' Demographic Data

\begin{tabular}{|c|c|c|c|c|}
\hline Patients' characteristics & Group $1(n=16)$ & Group $2(n=11)$ & Group $3(n=4)$ & $P$ value \\
\hline Age & $49.7 \pm 11$ & $28.8 \pm 12.2$ & $45.0 \pm 13.6$ & 0.54 \\
\hline Sex & $M=14, F=2$ & $\mathrm{M}=8, \mathrm{~F}=3$ & $\mathrm{M}=3, \mathrm{~F}=1$ & 0.400 \\
\hline \multirow{3}{*}{ Type of IBD } & $\mathrm{UC}=11$ & $\mathrm{UC}=6$ & $\mathrm{UC}=3$ & \multirow{3}{*}{0.01} \\
\hline & $\mathrm{CD}=2$ & $\mathrm{CD}=2$ & $\mathrm{CD}=1$ & \\
\hline & $\mathrm{IC}=3$ & $\mathrm{IC}=3$ & $\mathrm{IC}=0$ & \\
\hline \multirow{6}{*}{ Extend of disease } & Pancolitis $=4$ & Pancolitis $=2$ & Pancolitis $=0$ & \multirow{6}{*}{0.52} \\
\hline & Left sided $=7$ & Left sided $=5$ & Left sided $=2$ & \\
\hline & Proctitis $=2$ & Proctitis $=2$ & Proctitis $=1$ & \\
\hline & Colonic $\mathrm{CD}=1$ & Colonic $\mathrm{CD}=1$ & Colonic $\mathrm{CD}=0$ & \\
\hline & Ileal $=0$ & Ileal $=0$ & Ileal $=0$ & \\
\hline & Ileo-colonic $=2$ & Ileo-colonic $=1$ & Ileo-colonic $=1$ & \\
\hline Duration of disease & Mean $=13$ yrs. & Mean $=11 \mathrm{yrs}$ & Mean $=9$ yrs & 0.008 \\
\hline Hospital admission & 16 & 11 & 4 & 0.06 \\
\hline Steroids & 16 & 11 & 4 & 0.60 \\
\hline Thiopurine & 8 & 5 & 3 & 0.54 \\
\hline Antibiotics & 4 & 1 & 3 & 0.24 \\
\hline Anti TNF & 6 & 1 & 1 & 1.00 \\
\hline 5 ASA & 12 & 9 & 3 & 0.200 \\
\hline Cyclosporine & 3 & 0 & 1 & 1.00 \\
\hline Positive serology & 14 & 9 & 4 & 0.001 \\
\hline Previous surgery & 5 & 2 & 1 & 0.600 \\
\hline Positive cytopathic changes on $\mathrm{HE}$ & 13 & 8 & 3 & 0.46 \\
\hline $\begin{array}{l}\text { Colectomy } \\
\text { (three patients with UC had colectomy without } \\
\text { treatment with ganciclovir and were excluded) }\end{array}$ & 1 & 3 & 1 & 0.190 \\
\hline Death & 0 & 1 & 0 & 1.00 \\
\hline Transfusion reaction & 3 & 0 & 1 & 1.00 \\
\hline Side effect & 3 & 1 & 1 & 0.88 \\
\hline
\end{tabular}

UC- ulcerative colitis, CD- Crohn's disease, IC- Indeterminate colitis, TNF- tumor necrotic factor, ASA- aminosalicylic acid

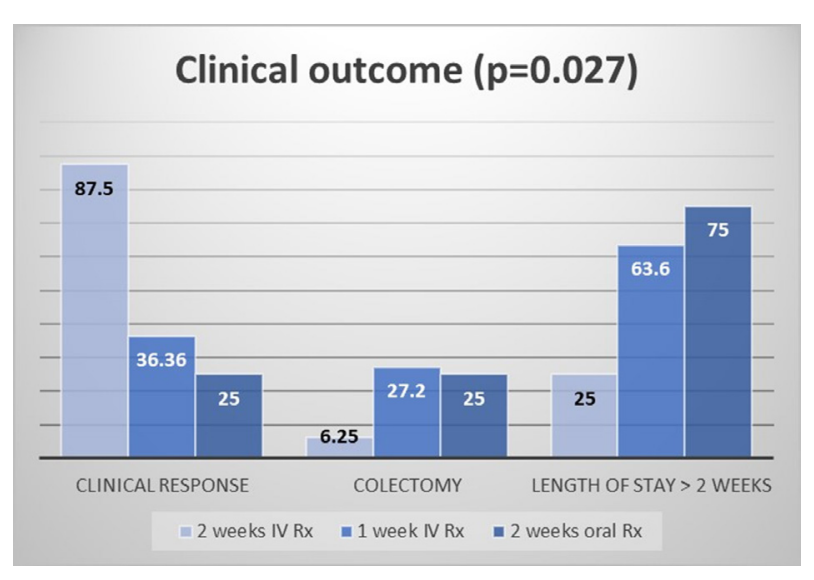

Fig.3: Graph showing clinical outcome, colectomy rate and length of stay (10 days) patients' characteristics and demographics data are shown in table 1.

In the colectomy section, three out of six patients with UC who had colectomy were excluded from the main analysis as they did not receive treatment with ganciclovir and the diagnosis of CMV was made later on the histological specimens.

All patients were on steroids for active IBD prior to starting ganciclovir. Eight patients $(\mathrm{UC}=5, \mathrm{CD}=3)$ also received anti TNF (tumor necrotic factor) treatment prior to commencing on ganciclovir. Clinical response was recorded as described above on day $3,7,10,14$, and 21 when available from the clinical records.

As described in figure 3, patients in group 1 (two weeks IV ganciclovir regime) were shown to do better 


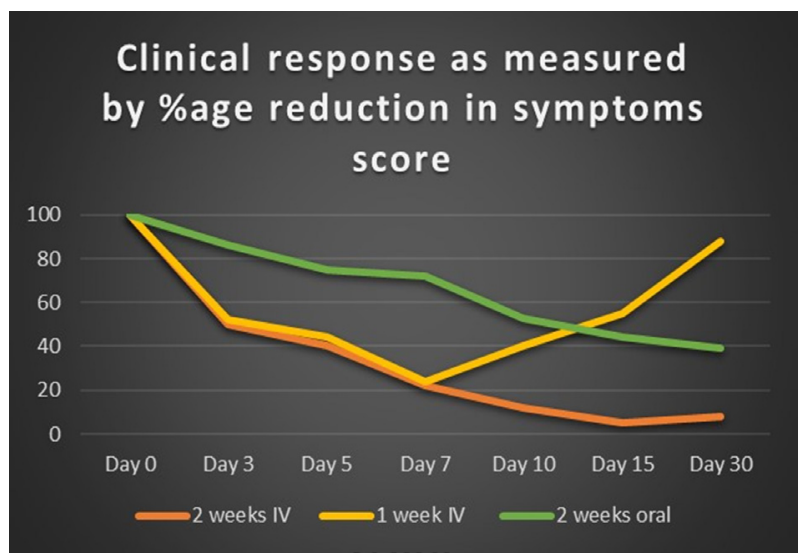

Fig.4: Graph showing clinical response in all three groups measured by percentage reduction in Harvey Bradshaw Index score on days $5,7,10$, and 15 .

compared with group 2 and group 3 (1 week of IV ganciclovir and 2 weeks of oral valganciclovir regimes) in terms of clinical response on week 2 (93.75\% vs $71.4 \%$, $25 \%$, respectively, $\mathrm{p}=0.867$ ). As shown by the graph in figure 4 , the clinical response rates on day 3 and 7 were similar in group 1 and group 2 as determined by improvement in symptoms score (64\% improvement on day 3 and $80 \%$ improvement on day 7) as compared with group 3 (14\% improvement on day 3 and 25\% improvement on day 7 ) but $54.6 \%$ of the patients in group 2 (6 out of 11 patients) had recurrence of symptoms within 4 weeks of stopping the treatment with IV ganciclovir and were re-admitted in hospital or reviewed by the healthcare physician requiring escalation of medical treatment in the form of steroids. 33.3\% of these patients (two out of six patients who had reoccurrence of symptoms) were tested for CMV re-infection and the results were negative. The remaining patients (four out of six patients) were not tested. Three out of these four patients (all with UC) deteriorated despite treatment and required colectomy. In group-1, 93.75\% of the patient achieved complete clinical response. $75 \%$ of the patients in group 1 (12 out of 16) were discharged from the hospital within 2 weeks of admission and the other 25\% (4) stayed more than 2 weeks, all were discharged within 3.5 weeks (figure 3). Two patients $(12.5 \%)$ in group 1 were readmitted within a month due to recurrence of symptoms, one of these responded with escalation of treatment while the other continued to deteriorate and required colectomy.

Overall, colectomy rate was higher $(100 \%)$ in those patients who did not receive the treatment with IV ganci-

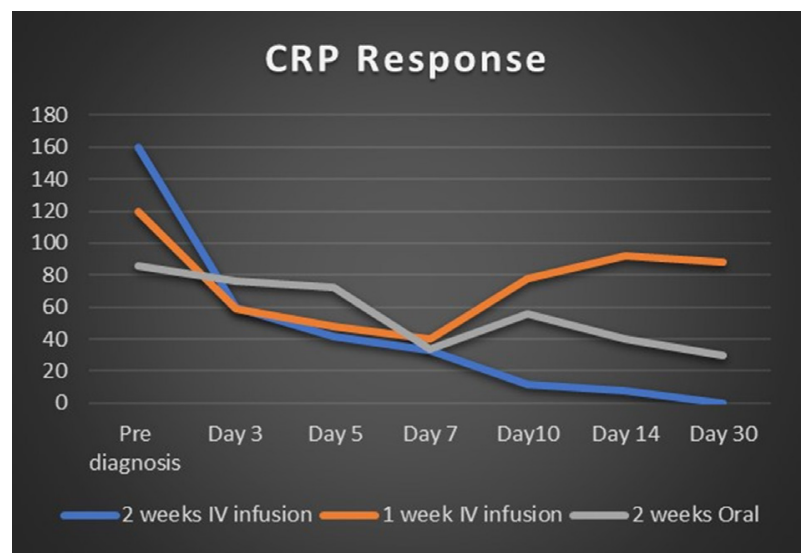

Fig.5: Graph showing CRP response in all three groups measured pre-treatment and post treatment up to day 30 .

clovir (all three patients with UC) compared with those who did $(16 \%, 4 / 31, p<0.01)$ as shown above in figure 2 .

As shown in figure-3, prolonged length of stay as inpatient in hospital (defined as more than 2 weeks) was observed in $25 \%$ in group $1,63.6 \%$ in group 2, and $100 \%$ in group 3 , respectively ( $p=0.027)$. Symptoms reoccurrence requiring re-admission and or escalation of treatment was lower in group 1 compared with other groups $(12.5 \%, 54.5 \%$, and $75 \%$, respectively, $p=0.045)$. The Colectomy rate in all three groups within 3 months are described by the graph in figure 3, which were $6.25 \%$ in group $1,27.3 \%$ in group 2 , and $25 \%$ in group 3 .

Symptoms severity score was recorded from the clinical notes against $\mathrm{HBI}$ index in patients with $\mathrm{CD}$ and SCCAI score in patients with UC and IC as described above. The pre-treatment symptoms score was considered as $100 \%$ and reduction in the symptoms score was recorded on day 3,5 , and 7 was similar in group 1 and group 2 compared with group 3. In group 1 and 2, symptoms score was reduced to $50 \%$ and $52 \%$ on day $3,40 \%$ and $42 \%$ on day 5 , and $20 \%$ and $24 \%$ on day 7 compared with group 3 ( $86 \%$ on day $3,75 \%$ on day 5 , and $72 \%$ on day 7 ). Patients in group 1 continued to improve with symptoms score of $12 \%$ on day 10 and $5 \%$ on day 15 and 30 . While in patients in group 2, symptoms were reoccurred after stopping the infusion with an increase of $40 \%$ in the symptom score on day 10 and $52 \%$ on day 15 . The patients in group 3 continued to have symptoms despite treatment $(53 \%$ on day 10 , and $44 \%$ on day 15$)$. These results however were not statistically significant as shown by the graph in figure-4.

As shown by the graphs in figure 5, CRP response 
rates on day 3 and 5, were superior in group 1 and 2 compared with group 3 but was non-significant $(P=0.67)$. The CRP response was found to be non-predictive but improvement in clinical symptoms on day 3 of treatment was found to be associated with better outcome. All the patients were tested for enteric infection and CDI via faecal analysis (ELISA) and none of the patients were positive for either enteric infection or CDT. Eight out of 31 patients (group- $1=4$, group- $2=1$, group- $3=3$ ) also received antibiotic in the form of metronidazole, ciprofloxacin, and cefotaxime in the first 3-5 days of admission for suspected infection. Two out of these patients were on cefotaxime for lower respiratory tract infection during hospital stay. One death was recorded but detailed clinical note investigation revealed that this was due to other co-morbidities (predominantly cardio-respiratory complication) and was not directly related to CMV colitis. Acute infusion reactions were defined as an adverse event that occurred within $1 \mathrm{~h}$ after infusion. Delayed hypersensitivity reactions were defined as the occurrence of myalgias, arthralgias, fever, or rash occurring 1-14 days after ganciclovir treatment. Three patients in group 1 and one patient of group 3 experienced mild severity of acute transfusion reaction. These were dealt by stopping the infusion immediately after the initial symptoms and clinical signs of infusion reaction and were treated by administering IV steroids $100 \mathrm{mg}$ stat and anti-histamine (chlorpheniramine $10 \mathrm{mg}$ IV). The infusion was later recommenced at a slower rate with close monitoring of the patients. These patients were discharged with clear instruction to report to Emergency Department on reoccurrence of any symptoms of delayed allergy reaction. Two patients reported mild delayed type of infusion reaction in term of headaches and myalgia, which were managed with simple analgesics with full recovery. Additionally, three patients in group 1 and one patient each from group 2 and 3 reported side effects in term of joint pain, sore throat, eye pain, hair fall, and body aches. There was patchy documentation about some patient that these side effects were dealt with symptomatic treatment in the form of analgesia.

\section{DISCUSSION}

The morbidities associated with IBD are debilitating with poor quality of life, prolonged hospital stay, and overall cost of treatment. Although it is widely acceptable that the course of disease and prognosis is significantly affected by concurrent CMV infection, in the absence of dedicated studies, the opinion about the impact of CMV infection over the course of IDB remains debatable. ${ }^{16}$ While some reviews suggested it to be a bystander in patients with IBD with no significant deleterious effect on the course of the disease, ${ }^{17}$ other studies, albeit small, showed that CMV colitis could aggravate the course of pre-existing IBD, increase its refractoriness to IBD therapy, and also increases the frequency of complications leading to increased colectomy rate. ${ }^{18,19}$ It is widely accepted that outcome of patients with concurrent CMV infection in IBD is different in those who were treated with antiviral therapy additional to IBD treatment compared with those who did not receive any anti-viral therapy. ${ }^{20,22}$ For example, a study by Mariguela and colleagues reviewed a population of patients with UC and found a statistically significant higher proportion of patients with enteric tissue CMV, $57.1 \%$ in UC compared with $14.3 \%$ in patients with colorectal cancer. ${ }^{23}$ Similarly, Nguyen and co-workers ${ }^{24}$ concluded that there was more reduction in the number of colectomies performed for patients treated with antiviral agents compared with those who did not receive anti-viral therapy. In support of this, another study by Roblin and colleagues ${ }^{25}$ compared the outcome of patients with IBD and CMV infection with and without anti-viral treatment. The researchers found that early use of antiviral treatment delayed the occurrence of resistance to steroids and other immunomodulatory therapies. On the other hand, a small study by Zeki and others reviewed the outcome of 17 patients with UC and concurrent diagnosis of CMV. This study (presented in an abstract form) found no difference in the colectomy rate in those who were treated for CMV infection compared with those who were not. ${ }^{26}$ In another study, Matsuoka and co-workers reviewed the outcome of 69 patients with active UC and concurrent diagnosis of CMV $(n=48)$ compared with those who did not have CMV infection $(\mathrm{n}=21)$. The outcome in term of rates of remission and colectomy were not significantly different among the CMV reactivation-positive, -negative, and CMV IgG negative groups. Furthermore, CMV disappeared without treatment with ganciclovir in most of the CMV reactivation-positive patients. ${ }^{27}$ In our study, three 
patients with severe UC who failed medical treatment with steroids and anti TNF and required colectomy, were later found to have CMV inclusion bodies in the colectomy specimens. These patients were not tested for CMV in the beginning and whether earlier testing and treatment for CMV with ganciclovir would have made any difference in the outcome is beyond from any conclusion.

CMV reactivation can also occur in immunocompetent patients, mostly resulting in a condition similar to the primary infection but rarely progress to a serious systemic illness. It can also occur in immunocompromised patients (elderly, under chemotherapy or immunosuppressive treatments, AIDS), in whom it can cause a more problematic course with a more serious systemic involvement. Several diagnostic modalities such as histology including immunohistochemistry, serology, CMV culture, PCR for CMV genome and CMV antigenemia have been suggested to confirm CMV infection. ${ }^{28,29,30}$ Endoscopic findings are essentially non-discriminatory and unable to diagnose the presence of CMV infection in patients with IBD. ${ }^{31,32,33}$ Few small studies however have suggested that presence of deep punched out liner ulceration may favor the presence of CMV infection in addition to active IBD but this has not been supported by larger series. ${ }^{34,2}$ In our study, diagnosis of CMV was made by detection of inclusion bodies in biopsy specimens from the colonic mucosa using hematoxylin and eosin (H\&E) staining or immunohistochemistry (IHC) using anti-CMV monoclonal antibodies. In most cases, CMV viral status was also confirmed by PCR.

Despite the conflicting opinion regarding the treatment of CMV infection on the background of IBD, ganciclovir has been proposed and traditionally used for the treatment both in IV as well as oral form. Although there was no conclusive data regarding the use, mode of administration, and duration of treatment with ganciclovir in CMV colitis, some small studies have suggested ganciclovir in IV form with a standard dosing regimen of $5 \mathrm{mg} / \mathrm{kg}$ IV every $12 \mathrm{~h}$ for $1-3$ weeks with a possible switch to oral ganciclovir $(1 \mathrm{~g} / 8 \mathrm{~h})$ after clinical improvement for the reminder of the course. ${ }^{17,35}$ In a study, Kim and others focused on IV ganciclovir as the antiviral agent most effective in patients with moderate to severe, steroid-refractory ulcerative colitis. In some other studies, it was found that the early start of antiviral therapy, with discontinuation or tapering of immunosuppressive therapy, could improve the prognosis particularly in patients with UC than in CD. ${ }^{22}$ This dosing regimen is widely acknowledged as the regimen of choice for treating CMV when associated with IBD. Our results were also in line with these studies because the use of IV ganciclovir has been shown to be more beneficial than oral ganciclovir ${ }^{36}$ due to its increased bioavailability; hence when giving ganciclovir IV one can expect an added benefit compared with oral medication. On the other hand, valganciclovir has been proven to be as adequate in CMV infection treatment in patients after transplantation as IV ganciclovir, but its efficacy regarding CMV colitis on the background of IBD has not been studied. ${ }^{37}$ These findings tend to go in line with the results encountered in our study, where patients treated with 2 weeks of IV ganciclovir showed more favorable outcome regarding hospital discharge, shortened length of stay, and low colectomy rate. For example, patients who were started on 2 weeks of IV ganciclovir showed an overall improvement in clinical outcome as well as an overall decrease in colectomy rate and duration of stay in the hospital when compared with patients who received oral valganciclovir or one week of IV ganciclovir. The disease reoccurrence rate was higher in those patients who received only one week of IV ganciclovir (54.6\% vs $12.5 \%$ ) compared with the group who received 2 weeks of IV infusion. Colectomy rate was also higher in those with recurrence of disease after stopping treatment with IV ganciclovir. This reflects the inadequacy of one week IV infusion in these patients.

There was a noted decrease in CRP levels starting from day 3 of IV treatment compared with patients who were on different medication regimens. Despite the decreasing levels, this proved not to be an adequate predictor of a better outcome for our patient population. We also had a concern that there would be an increased risk of Clostridium difficile infection (CDI) but our study proved it to be false as none of the patients included in the study was tested positive for CDI. It is worth noting that the correlation of CDI and CMV colitis has not been adequately studied in the literature.

A total of eight patients in the study (four in group 1, 
one in group 2, and three in group 3) received antibiotic. Tree of these patients received antibiotic as prophylaxis for pneumocystis carinii pneumonia (PCP) being on three type of immunosuppression (anti TNF, thiopurine, and steroids), two patients received antibiotic for lower respiratory tract infection, two for urinary tract infection, and one patient received antibiotic for suspected infective colitis initially, which was stopped after 48 hours on confirmation of clear stool specimen. None of these patients received antibiotic for complication of colitis such as toxic megacolon or perforation.

In summary, there is a complexity in the association of IBD to CMV, and recently, more and more observations are made pointing out that the appropriate use of effective antiviral drugs, would more probably regain the sensitivity to steroids and immunomodulatory therapies and reduce the risk of colectomy with production of favorable outcome in patients with moderate to severe IBD. There are still needs to large randomized trials to emphasize these observations.

\section{CONCLUSION}

CMV colitis is associated with poor outcome in patient with IBD if left untreated. Data regarding mode and duration of treatment remain poorly defined. In our experience 2 weeks of IV ganciclovir was associated with a better outcome. Little or no improvement in the clinical condition on day 3 of treatment was associated with colectomy. Further data are required to evaluate the treatment guidance of this condition.

\section{ACKNOWLEDGEMENT}

The authors acknowledge the support and help from the Pharmacy, Histopathology, and Microbiology Departments, Royal United Hospital Bath and North Bristol Hospital NHS Trust for provision of information.

\section{ETHICAL APPROVAL}

There is nothing to be declared.

\section{CONFLICT OF INTEREST}

The authors declare no conflict of interest related to this work.

\section{REFERENCES}

1. Kotton CN. CMV: Prevention, Diagnosis and Therapy. Am J Transplant 2013;13 Suppl 3:24-40. doi:10.1111/ ajt.12006.

2. Rowshani AT, Bemelman FJ, van Leeuwen EM, van Lier RA, Ten Berge IJ. Clinical and immunologic aspects of cytomegalovirus infection in solid organ transplant recipients. Transplantation 2005;79:381-6. doi:10.1097/01. TP.0000148239.00384.F0.

3. You DM, Johnson MD. Cytomegalovirus infection and the gastrointestinal tract. Curr Gastroenterol Rep 2012;14:334-42. doi:10.1007/s11894-012-0266-4.

4. Kandiel A, Lashner B. Cytomegalovirus colitis complicating inflammatory bowel disease. Am J Gastroenterol 2006;101:2857-65. doi:10.1111/j.1572-0241.2006.00869.x.

5. Kim JJ, Simpson N, Klipfel N, Debose R, Barr N, Laine L. Cytomegalovirus infection in patients with active inflammatory bowel disease. Dig Dis Sci 2010;55:1059-65. doi:10.1007/s10620-010-1126-4.

6. Maher MM, Nassar MI. Acute cytomegalovirus infection is a risk factor in refractory and complicated inflammatory bowel disease. Dig Dis Sci 2009;54:2456-62. doi: 10.1007/s10620-008-0639-6.

7. Hommes DW, Sterringa G, van Deventer SJ, Tytgat GN, Weel J. The pathogenicity of cytomegalovirus in inflammatory bowel disease: a systematic review and evidencebased recommendations for future research. Inflamm Bowel Dis 2004;10:245-50. doi:10.1097/00054725-20040500000011.

8. Criscuoli V, Casà A, Orlando A, Pecoraro G, Oliva L, Traina M, et al. Severe acute colitis associated with CMV: a prevalence study. Dig Liver Dis 2004;36:818-20. doi:10.1016/j.dld.2004.05.013.

9. Matsumoto S, Yoshida Y. What are the factors that affect hospitalization and surgery for aggravation of ulcerative colitis? Eur J Gastroenterol Hepatol 2014;26:282-7. doi: 10.1097/MEG.0000000000000028.

10. Goodman AL, Murray CD, Watkins J, Griffiths PD, Webster DP. CMV in the gut: a critical review of CMV detection in the immunocompetent host with colitis. Eur J Clin Microbiol Infect Dis 2015;34:13-8. doi:10.1007/s10096014-2212-x

11. Kotton CN, Kumar D, Caliendo AM, Asberg A, Chou S, Danziger-Isakov L, et al. Updated international consensus guidelines on the management of cytomegalovirus in solid-organ transplantation. Transplantation 2013;96:33360. doi:10.1097/TP.0b013e31829df29d. 
12. de la Hoz RE, Stephens G, Sherlock C. Diagnosis and treatment approaches of CMV infections in adult patients. J Clin Virol 2002;25 Suppl 2:S1-12.

13. Subramanian V, Finlayson C, Harrison T, Rice P, Pollok R. Primary cytomegalovirus infectious colitis complicating Crohn's disease successfully treated with oral valganciclovir. J Crohns Colitis 2010;4:199-202. doi:10.1016/j. crohns.2009.11.004.

14. Kim YS, Kim YH, Kim JS, Cheon JH, Ye BD, Jung SA, et al. The prevalence and efficacy of ganciclovir on steroidrefractory ulcerative colitis with cytomegalovirus infection: a prospective multicenter study. J Clin Gastroenterol 2012;46:51-6. doi:10.1097/MCG.0b013e3182160c9c.

15. European evidence-based Consensus on the prevention, diagnosis and management of opportunistic infections in IBD, ECCO guidelines, 2009.

16. Papadakis KA, Tung JK, Binder SW, Kam LY, Abreu MT, Targan SR, et al. Outcome of cytomegalovirus infections in patients with inflammatory bowel disease. Am J Gastroenterol 2001;96:2137-42.

17. Lawlor G, Moss AC. Cytomegalovirus in inflammatory bowel disease: pathogen or innocent bystander? Inflamm Bowel Dis 2010;16:1620-7. doi:10.1002/ibd.21275.

18. Römkens TE, Bulte GJ, Nissen LH, Drenth JP. Cytomegalovirus in inflammatory bowel disease: A systematic review. World J Gastroenterol 2016;22:1321-30. doi: 10.3748/wjg.v22.i3.1321.

19. Sager K, Alam S, Bond A, Chnnappan L, Probert CS. Review article: cytomegalovirus and inflammatory bowel disease. Aliment Pharmacol Ther 2015;41:725-33. doi: 10.1111/apt.13124.

20. Kim YS, Kim YH, Kim JS, Jeong SY, Park SJ, Cheon JH, et al. Long-term outcomes of cytomegalovirus reactivation in patients with moderate to severe ulcerative colitis: a multicenter study. Gut Liver 2014;8:643-7. doi: 10.5009/ gnl13427.

21. Maher MM, Nassar MI. Acute cytomegalovirus infection is a risk factor in refractory and complicated inflammatory bowel disease. Dig Dis Sci 2009;54:2456-62. doi: 10.1007/s10620-008-0639-6.

22. Park SH, Yang SK, Hong SM, Park SK, Kim JW, Lee HJ, et al. Severe disease activity and cytomegalovirus colitis are predictive of a nonresponse to infliximab in patients with ulcerative colitis. Dig Dis Sci 2013;58:3592-9. doi: 10.1007/s10620-013-2828-1.

23. Mariguela VC, Chacha SG, Cunha AA, Troncon LE, Zucoloto S, Figueiredo LT. Cytomegalovirus in colorec- tal cancer and idiopathic ulcerative colitis. Rev Inst Med Trop Sao Paulo 2008;50:83-7. doi:10.1590/S003646652008000200004

24. Nguyen M, Bradford K, Zhang X, Shih DQ. Cytomegalovirus reactivation in ulcerative colitis patients. Ulcers 2011;2011. doi:10.1155/2011/282507.

25. Roblin X, Pillet S, Oussalah A, Berthelot P, Del Tedesco E, Phelip JM, et al. Cytomegalovirus load in inflamed intestinal tissue is predictive of resistance to immunosuppressive therapy in ulcerative colitis. Am J Gastroenterol 2011;106:2001-8. doi:10.1038/ajg.2011.202.

26. Zeki SS, Kodati S, Jordan A, Hansi N, Thomas-Gibson S, Nightingale JM. Colectomy rates for patients treated for CMV disease in the context of Ulcerative Colitis do not differ from those who are not treated for CMV disease. Gastroenterology 2010;138:S-537.

27. Matsuoka K, Iwao Y, Mori T, Sakuraba A, Yajima T, Hisamatsu $\mathrm{T}$, et al. Cytomegalovirus is frequently reactivated and disappears without antiviral agents in ulcerative colitis patients. Am J Gastroenterol 2007;102:331-7. doi:10.1111/j.1572-0241.2006.00989.x.

28. de la Hoz RE, Stephens G, Sherlock C. Diagnosis and treatment approaches of CMV infections in adult patients. $J$ Clin Virol 2002;25 Suppl 2:S1-12. doi:10.1016/S13866532(02)00091-4.

29. Meyers JD, Ljungman P, Fisher LD. Cytomegalovirus excretion as a predictor of cytomegalovirus disease after marrow transplantation: importance of cytomegalovirus viremia. J Infect Dis 1990;162:373-80. doi:10.1093/ infdis/162.2.373.

30. Boivin G, Handfield J, Toma E, Murray G, Lalonde R, Tevere VJ, et al. Evaluation of the AMPLICOR cytomegalovirus test with specimens from human immunodeficiency virus-infected subjects. J Clin Microbiol 1998;36:2509-13.

31. Wada Y, Matsui T, Matake H, Sakurai T, Yamamoto J, Kikuchi Y, et al. Intractable ulcerative colitis caused by cytomegalovirus infection: a prospective study on prevalence, diagnosis, and treatment. Dis Colon Rectum 2003;46:S5965. doi:10.1097/01.DCR.0000087486.21981.C6.

32. Sakamoto I, Shirai T, Kamide T, Igarashi M, Koike J, Ito A, et al. Cytomegalovirus enterocolitis in an immunocompetent individual. J Clin Gastroenterol 2002;34:243-6.

33. Suzuki H, Kato J, Kuriyama M, Hiraoka S, Kuwaki K, Yamamoto K. Specific endoscopic features of ulcerative colitis complicated by cytomegalovirus infection. World $J$ Gastroenterol 2010;16:1245-51.

34. Nishimoto Y, Matsumoto T, Suekane H, Shimizu M, Mi- 
kami Y, Iida M. Cytomegalovirus infection in a patient with ulcerative colitis: colonoscopic findings. Gastrointest Endosc 2001;53:816-8. doi:10.1067/mge.2001.114955.

35. Paya C, Humar A, Dominguez E, Washburn K, Blumberg E, Alexander B, et al. Efficacy and Safety of Valganciclovir vs. Oral Ganciclovir for Prevention of Cytomegalovirus Disease in Solid Organ Transplant Recipients. Am J Transplant 2004;4:611-20. doi:10.1111/j.16006143.2004.00382.x.

36. Åsberg A, Humar A, Rollag H, Jardine AG, Mouas H, Pescovitz MD, et al. Oral Valganciclovir Is Noninferior to Intravenous Ganciclovir for the Treatment of Cytomegalovirus Disease in Solid Organ Transplant Recipients. Am J Transplant 2007;7:2106-13. doi:10.1111/j.16006143.2007.01910.x.

37. Åsberg A, Humar A, Jardine AG, Rollag H, Pescovitz MD, Mouas H, et al. Long-Term Outcomes of CMV Disease Treatment with Valganciclovir Versus IV Ganciclovir in Solid Organ Transplant Recipients. Am J Transplant 2009;9:1205-13. doi:10.1111/j.1600-6143.2009.02617.x. 